\section{Perception of two-dimensional Markov constraints within visual displays*}

\author{
IRWIN POLLACK $\dagger$ \\ University of Michigan, Ann Arbor, Michigan 41804
}

A method is described for constructing visual displays in which statistical constraints are encoded within two spatial dimensions without introducing one-dimensional linear constraints. Within each local group of four elements, the state of one element was determined with a given probability by the previously generated states of the other three. Ss rated such displays on a scale from "lumpy" (or crude texture) to "lacy" (or even texture). The consistency of classification obtained for displays with strong aggregated ("lumpy") properties was substantially higher than that obtained for displays with strong distributed ("lacy") properties. An incidental feature of the Ss' behavior was their deliberate degrading of the visual quality of the displays. Comparison is made with one-dimensional displays concatenated in two dimensions.

The sensitivity of $\mathrm{Ss}$ to statistically encoded information may be examined: (1) on a "cognitive" basis by the probability-guessing experiment in which successive trials are presented at the rate of about one trial per $10 \mathrm{sec}$; or (2) on a "perceptual" basis by encoding statistical information in the form of a display, and then presenting many items in a short period of time. The former method has the advantage that responses can be obtained after each item of a sequence. The latter method has the advantage of efficiency, and is well illustrated by a study of Picket $t$ (1964). He controlled the second-order probabilities of a Markov generator of dots and spaces. When the probability of either element's repeating itself was high, a "lumpy" linear string of characters was generated. Groups of successive dots were separated by groups of successive spaces. When the probability of repetition of an element was low, an "evenly spaced" string of elements was generated. Dots and spaces alternated. Thus, manipulations of the conditional repetition probability were translated in terms of the texture of the display. Julesz (1962) had earlier produced displays differing in texture by the same general procedure. The role of textural differences in the perception of surfaces has been vigorously championed by Gibson (1950).

In the Pickett and Julesz displays successive lines of the display were

*The writer is indebted to Mr. Robert Hsieh, for the computer program, for the displays, and for analyzing the results, and to Mrs. K. Weidner, for supervising the experimental tests. Computer time was provided by the Computational Center, University of Michigan Medical School. The research was supported in part by $\mathrm{National}$ Science Foundation Grant GB 6148

TMailing address: Mental Health Research Institute, University of Michjgan, Ann Arbor. Michigan 48104. generated independently of each other. The independently derived, one-dimensional linear strings were concatenated to yield textural differences for two-dimensional displays. Julesz (1962, Fig. 8) also demonstrated a procedure for developing displays with two-dimensional constraints, based upon clustering within groups of three elements to provide a varying "granularity."

The present study extends the Pickett-Julesz approach to displays with two-dimensional textural properties. The statistical restrictions reside entirely within two-dimensional constraints by means of a procedure with consequences substantially different from Julesz's procedure. The primary aim was to determine if such constraints can, indeed, be perceived in the absence of one-dimensional constraints.

\section{DISPLAYS WITH TWO-DIMENSIONAL SPATIAL CONSTRAINTS \\ Method of Generation}

Two dimensional Markov displays were generated by the following rules. The top row and the first column of a 54 by 54 element display were independently generated without sequential restriction:!

$$
\begin{aligned}
P(X \mid X)=P(0 \mid 0) & =P(X \mid 0) \\
& =P(0 \mid X)=0.50
\end{aligned}
$$

where $X$ represents the $X$ symbol and 0 represents a space. Every other position of the display was generated by a process illustrated in Fig. 1.

Assume that the elements at the northwest, northeast, and southwest corners of a set of four elements have been specified. The to-be-determined target element in the southeast corner is determined by the configuration of its immediate neighbors. The selection rule is that the aimed-for sum for each group of four elements is even, with probability $p$, where $X$ and 0 are represented by the binary elements, zero and one. Specifically, the southeast target element repeats the element in the northwest corner with probability $p$ for Patterns 1-4, and repeats the complement of the element in the northwest corner with probability $p$ for Patterns 5-8. In Patterns 1-4, the elements in the northeast and southwest corners are identical; in Patterns 5-8, these elements are different. The effect of this rule, for large $p$ values, is to encourage an even number of each element within each square, and, especially, a clumping of identical elements for Patterns 2 and 4. The effect of this rule, for low $p$ values, is to encourage an odd number of each element within each square, and, especially, a break-up of the clumping of identical elements for Patterns 2 and 4.

It is noted that each northwest-northeast horizontal sequence is associated with each target element with probability p. For example, the sequence $0 X$ in Patterns 1 and 5 is paired both with 0 and $X$ in the southeast position. Each northwest-southwest vertical sequence is also paired with each target element with probability $p$, as 0 over $X$ in Patterns 1 and 8. Similarly, each individual element in the northwest, northeast, and southwest positions is paired equally of ten with each target element in the southeast position, as $X$ in the northeast position in Patterns 1,2, 5 , and 6 , or as 0 in the southwest position in Patterns 3, 4, 5, 6. Thus, the initial conditional probabilities remain at 0.50 for the horizontal, vertical, and diagonal dimensions, separately. It is only with the two-dimensional contingencies that there obtains departures from the marginal probabilities.

With Positions $(1,1)(1,2)$ and $(2,1)$ determined by the independent generation of the top row and first column, the element at Position $(2,2)$ was next determined by random sampling, with defined probability $p$. The element at Position $(3,2)$ was next determined with

$$
\text { IWO-DIMENSIONAL LOCAL FEATURES }
$$

$\begin{array}{cccc}1 & 3 & 5 & 7 \\ 0 \times & \times 0 & 0 \times & \times 0 \\ \times 0^{*} & 0 x^{*} & 0 \times 0^{*} & \times 0^{*} \\ 2 & 4 & 6 & 8 \\ \times \times & 00 & \times \times & 00 \\ \times x^{*} & 00^{*} & 00^{*} & \times x^{*}\end{array}$

Fig. 1. Local features of displays with two-dimensional constraints. An asterisk indicates that the southeast corner was selected with probability $p$. 
displays. Successive intervals on the $\mathrm{x}$-axis represent displays of successive conditions of decreasing $p$ values. In terms of the scale

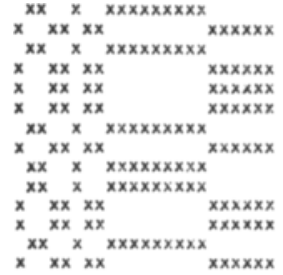

.9999

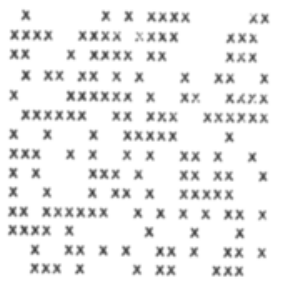

$P(A \mid A)$

.5000

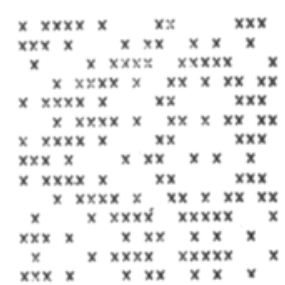

$P(A \mid A)$

.0001

\begin{tabular}{|c|c|c|c|c|}
\hline & & $x x$ & $x \times x$ & $x \times x$ \\
\hline$x x$ & $x \quad x x$ & $x x$ & $x \times x$ & $x \times x$ \\
\hline$x x$ & $x \times x$ & $x x$ & $x \times x$ & $x \times x$ \\
\hline$x x$ & $x \quad x x$ & $x x$ & $x \times x$ & $x \times x$ \\
\hline$x x$ & $x \times x$ & $x x$ & $x \times x$ & $x \times x$ \\
\hline$x x$ & $x \quad x x$ & $x x$ & $x=x$ & $x \times x$ \\
\hline$x x$ & $x \times x$ & $x x$ & $x \times x$ & $x \times x$ \\
\hline$x x$ & $x \quad x x$ & $x x$ & $x \times x$ & $x \times x$ \\
\hline$x x$ & $x \quad x x$ & $x x$ & $x \times x$ & $x \times x$ \\
\hline$x x$ & $x \quad x x$ & $x x$ & $x \times x$ & $x \times x$ \\
\hline$x x$ & $x \times x$ & $x \mathbf{x}$ & $x \times x$ & $x \times x$ \\
\hline$x x$ & $x x$ & $x x$ & $x \times x$ & $2 \times x$ \\
\hline$x x$ & $x \quad x x$ & $x x$ & $x \times x$ & $x \times x$ \\
\hline$x$ & $x x$ & $x x$ & $x \times x$ & $x \times x$ \\
\hline
\end{tabular}

of $p$ values, the change in $p$ as $p(1-p)$ is changed from .01 to .02 is about 0.01 ( 0.9898 vs 0.9796 for Ranks 1 and 2 and 0.0204 vs 0.0102 for Ranks 49 and 50 ); the change in $p$ as $p(1-p)$ is changed from .24 to .25 is $0.10(0.60$ vs 0.50 for Display Ranks 24 and 25 and 0.50 vs 0.40 for Ranks 26 and 27). The $y$-axis represents assigned ratings by the Ss. The numerical entries in Fig. 3 represent the number of Ss who assigned the same rating to a given display, the period entry represents a single $S$, and the colon entry represents two Ss who assigned the same rating to a given display. The entries in each column and row sum to 20, representing: in the case of columns, the distribution of assigned ratings assigned to a given display; and in the case of rows, the distribution of displays associated with a given assigned rating. The smooth lines represent the approximate median rating and the $12.5 \%-87.5 \%$ range of judgments.

Most notable is the relatively low scatter of assigned ratings for displays with low rank (with high $p$ values), as contrasted with the relatively high scatter of assigned ratings for displays with intermediate and high rank (with intermediate and low $p$ respect to Elements $(1,2) \quad(1,3)$ and generated.

Displays of 54 columns and 54 rows were generated by an IBM 1443 printer driven by an IBM 1800 computer. A range of $p$ values with $p(1-p)$ products of 0.01 , $0.02, \ldots, 0.25$ generated 50 displays: one for $p$ and one for $(1-p)$ at each of the 25 $p(1-p)$ products. Sample sections of the displays are presented in the top row of Fig. 2. The 54 by 54 display measured about $5.7 \times 8.9 \mathrm{~cm}$.

\section{Procedure}

Each display was inserted in a clear plastic holder for easy handling. Three displays at $p=.9999,0.50$, and .0001 were labeled as examples of most "lumpy," most "random," and most "lacy," respectively. The three displays were available for inspection at all times. Individual Ss rank-ordered the 50 displays from most lumpy through random to most lacy. The instructions suggested that the rating task would be easier after a preliminary crude classification was completed. Twenty university students served as Ss. One interesting feature of the behavior of several Ss is that they backed off from the displays, or tilted the displays at extreme viewing angles, especially for the judgment of lacy displays. While lumpy displays were often quickly perceived in terms of checkerboard clumps and holes, only several Ss expressed confidence in differentiating between the lacy and random displays.

\section{Results}

Figure 3 presents the individual judgments for the 50 two-dimensional

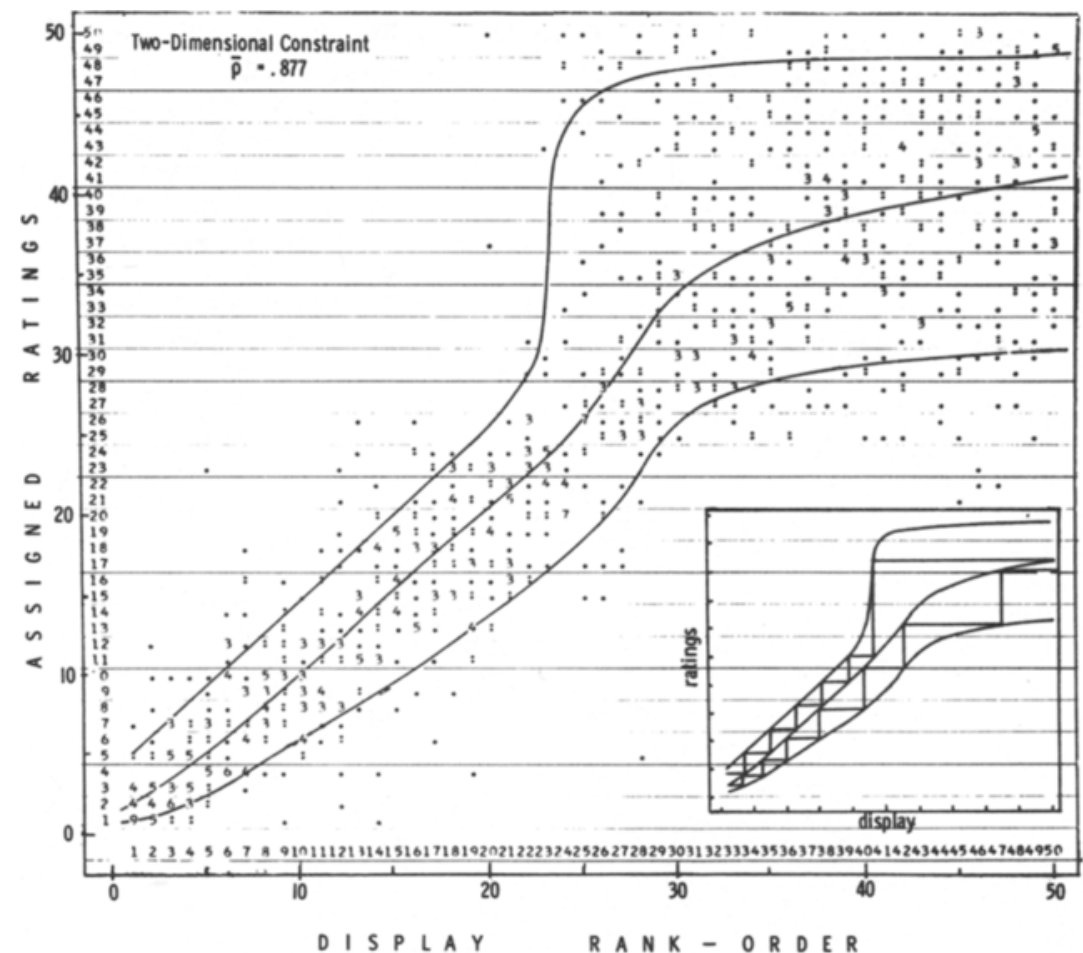

Fig. 3. Rank orderings of displays with two-dimensional constraints on a scale of most "lumpy" to most "lacy." The individual points represent the number of individual Os who assigned the same rating to a given display. The smooth curves mark the median ratings and the $12.5 \%-87.5 \%$ range. The insert illustrates the calculation of the number of discriminable steps. 
Table 1

Summary Measures of A seigned Ratings of Displays With Two- and OneDimensional Constraints

\begin{tabular}{ccc}
\hline & \multicolumn{2}{c}{ Dimensional Constraints } \\
\cline { 2 - 3 } Measure & Two & One \\
\hline Overall o & .877 & .978 \\
(Range) & $(.716-.970)$ & $(.956-.997)$ \\
Standard Deviation & 6.91 & 2.88 \\
(Range) & $(3.55-10.9)$ & $(1.18-4.44)$ \\
Absolute Deviation & 5.01 & 2.05 \\
(Range) & $(2.9-7.6)$ & $(.92-3.2)$ \\
Displays & & \\
$1-10$ & 2.48 & 1.10 \\
$11-20$ & 3.28 & 1.54 \\
$21-30$ & 5.39 & 1.67 \\
$31-40$ & 6.18 & 3.44 \\
$41-50$ & 7.73 & 2.48 \\
Correlation, & & \\
Displays & & \\
$1-10$ & .60 & .88 \\
$11-20$ & .58 & .83 \\
$21-30$ & .64 & .86 \\
$31-40$ & .13 & .43 \\
$41-50$ & -.06 & .73 \\
\hline
\end{tabular}

values). Stated otherwise, the "lumpy" displays were ranked consistently, but the various Ss could not clearly discriminate between "random" and "lacy" displays.

Table 1 presents summary statistics based upon the data of individual Ss. The rank-order correlation, the standard deviation of the differences between assigned and actual rankings, and the average absolute deviation were calculated for each of the 20 Ss. The average rank-order correlation (and the range over the $20 \mathrm{Ss}$ ), the average standard deviation (and the range), and the average absolute deviation (and the range) are presented. Also presented is the absolute deviation and the product-inoment correlation for each successive group of 10 display ranks, where 1-10 represent the lumpy displays and 41.50 represent the lacy displays. The individual entries are not entirely independent. For example, given the ratings for Displays 1.40 , the absolute deviation for Displays 41.50 is partly constrained.

Can we make any statements which might differentiate good from poor Ss (as defined by the overall rank-order correlation)? One notion is that all Ss will demonstrate relatively good performance for lumpy displays (low ranks) and that discrimination among $S s$ will be obtained primarily in terms of responses for random and lacy displays (intermediate and high ranks). Another notion is that good and poor Ss will be consistently differentiated for all displays. Table 2 presents the results for successive groups of five Ss, ordered in for the indicated displays. With two-dimensional displays, good and poor terms of the overall rank-order correlation,
Ss are consistently differentiated over all displays.

A rough measure of discriminability is obtained by the method schematically represented in the insert to Fig. 3. A graphic procedure is employed to determine how many "steps" of discriminability are revealed in terms of the variability of the ratings. It is assumed that displays are confusable to the extent to which they share the same assigned ratings. If there is little overlap of ratings, many discriminable steps are obtained; if greater overlap, few discriminable steps are obtained. A line from the median associated with Display Rank 1 is drawn parallel to the $x$-axis until it intersects with the lower bound, say at about Display Rank 4. This defines the first discriminable step. From the median associated with the first intersection, the procedure is again repeated. The procedure is then continued over the entire range of displays. Similarly, the procedure could start with Display 50 and proceed in the opposite direction. For the $12.5 \%-87.5 \%$ smoothed bounds in Fig. 3, only about seven discriminable steps are obtained. Of course, if the discriminability limits were lower, say $25 \% .75 \%$, a smaller number of discriminable steps would have been obtained.

\section{DISPLAYS WITH ONE-DIMENSIONAL SPATIAL CONSTRAINTS \\ Method of Generation}

The method of Pickett (1964) was employed to generate displays with one-dimensional constraints. The top row of a 54 by 54 element display was independently generated without sequential constraint. Successive positions within each of the columns were generated by repeating the previously generated element with probability $\mathrm{p}$. Thus, $\mathrm{P}(\mathrm{X} \mid \mathrm{X})$ $=\mathrm{P}(0 \mid 0)=\mathrm{p}$, and $\mathrm{P}(\mathrm{X} \mid 0)=\mathrm{P}(0 \mid \mathrm{X})=$ $(1-p)$. Samples of the displays are presented in the bottom row of Fig. 2 . Note that, although the display is two-dimensional, all constraints are only one-dimensional. The algorithm for Table 2
Tndividual

Individual Differences Associated With Assigned Ratings

*Each entry is the average absolute deviation between the assigned rating and the display ranking for the respective subgroup of $S s$ and displays. Chance deviation $=8:$ maximum deviation $=12$. also be stated in the same manner as that for two-dimensional displays: the aimed-for sum for each group of two successive units is even, with probability $p$.

\section{Procedure}

The procedure was identical with that of the two-dimensional tests previously described. Ten of the Ss first completed the one-dimensional tests; the other $10 \mathrm{Ss}$ first completed the two-dimensional tests. The three reference displays were labeled as most "striped," most "random," and most "thin."

\section{Results}

Figure 4 presents the individual judgments for the 50 one-dimensional displays. By comparison with the two-dimensional displays of Fig. 3 , the variability of ratings is sharply reduced, although performance is again consistently better for displays with the higher $\mathrm{p}$ values (the lower ranks). The summary of the individual results is presented in Table 2. In contrast with the findings in the two-dimensional tests, Ss were nearly of equal proficiency for one-dimensional displays of low rank (1-10), but were differentiated for displays of higher rank. The discriminability analysis illustrated in the insert yields about 20 discriminable steps-about three times the number yielded by the two-dimensional displays for the same range of $\mathrm{p}$ values.

Within the relatively homogeneous population of Ss employed, is there a correlation between proficiency on the one- and two-dimensional tests? Apparently, yes. The 20 Ss were ranked, in terms of the overall rank-order correlation, within the one-dimensional tests alone and within the two-dimensional tests alone. The rank-order correlation across the two sets of rankings was 0.76 .

Two-dimensional spatial constraints, in the display format studied in the present tests, are consistently less well

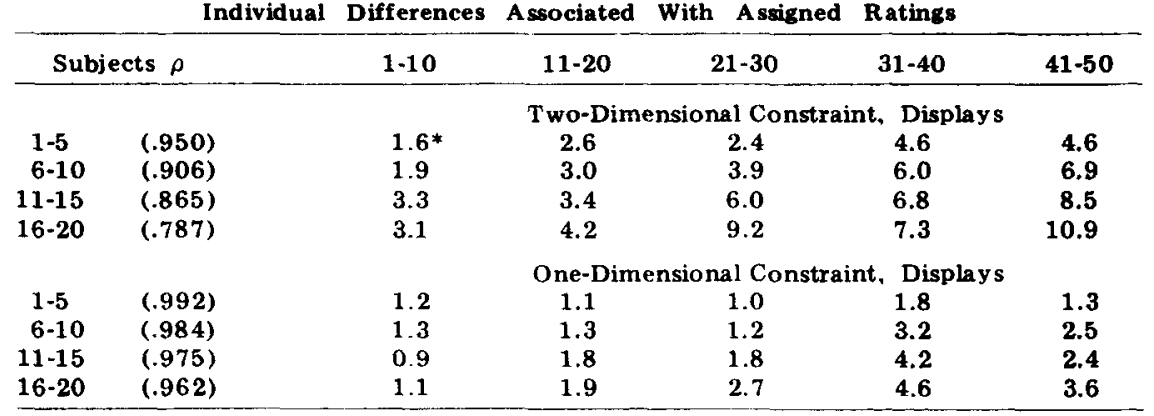

generating the one-dimensional display can 


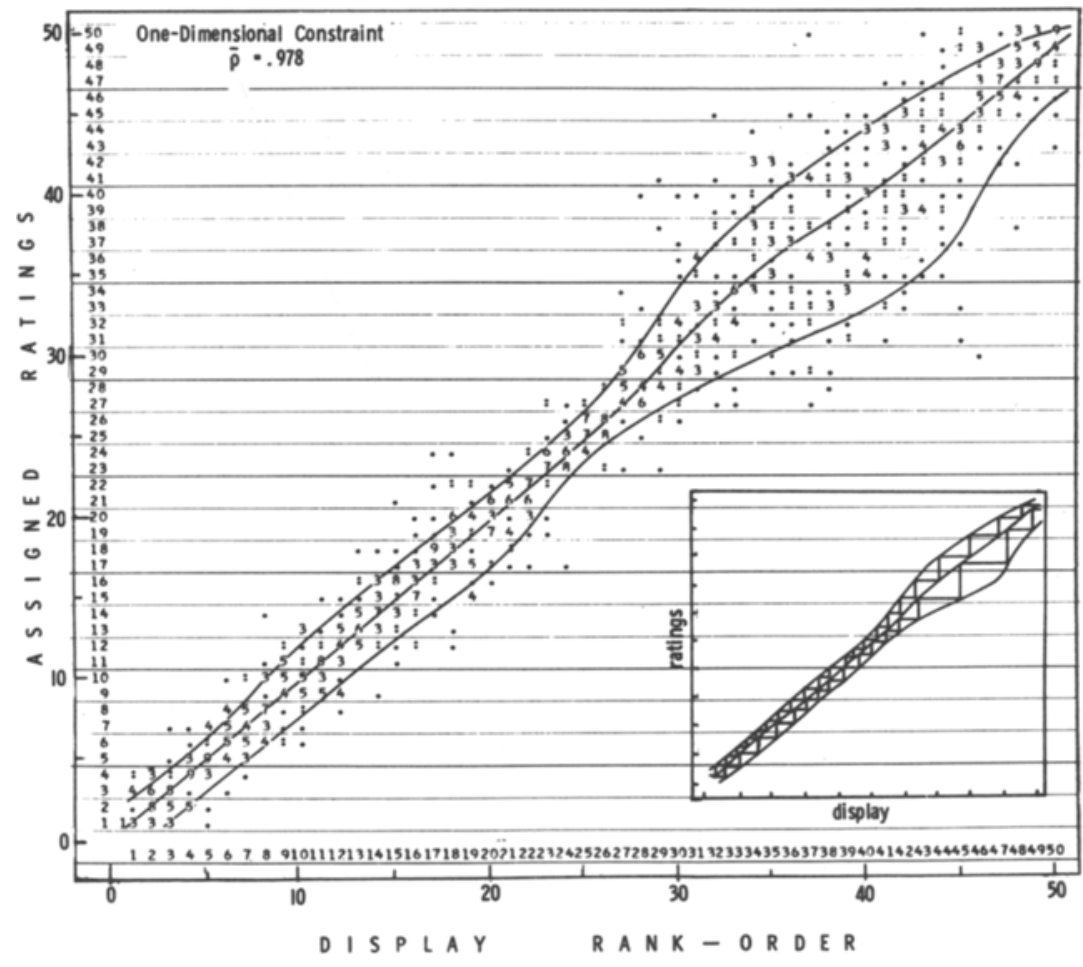

Fig. 4. As Fig. 3, for displays with one-dimensional constraints.

differentiated across Ss than one-dimensional spatial constraints. While the rating method does not easily yield well-defined classical thresholds, the several related measures are in agreement that two-dimensional spatial constraints can indeed be appreciated in the absence of one-dimensional constraints.

Why are the two-dimensional constraints so much harder to perceive? One hypothesis (generously supplied by an editorial referee) is that the one-dimensional constraints propagate themselves further in terms of repeated elements than do the two-dimensional constraints. For example, with a probability of an even aimed-for sum of 1.0 , the entire column of the one-dimensional display is uniform and is determined solely by the state of the initial element in that column. By contrast, with the two-dimensional display, the distance of propagation of repeated elements, i.e., the size of the checkerboard, is determined jointly by the two initial random strings.
A second, closely related hypothesis is that method of generating the two-dimensional constraints requires the specification of a greater number of random elements than do the one-dimensional constraints. A partial test of this hypothesis is to develop one- and two-dimensional displays with the same number of initial random elements. Preliminary tests suggest continued superior sensitivity for the one-dimensional displays. Moreover, in the extreme case where the outside column of the two-dimensional display employs only dots or only spaces, and where $\mathrm{p}=1.0$, the oneand the two-dimensional constraints will both result in the same display. This reasoning implies that the discriminability of two-dimensional constraints may be related to the conditional repetition probability associated with the initializing linear strings of the displays.

A third hypothesis (generously supplied by another editorial referee) relates to our poor understanding of the geometry of perceived visual space. The Markov generators developed concatenated linear strings or two-dimensional rectangular displays. On the other hand, the internal scanning of the human visual system is most likely neither serial nor linear nor rectangular. The present Markov constraints may, therefore, be completely meaningless from a perceptual point of view. Nevertheless, the one-dimensional constraints can probably be topologically transformed more easily into the idealized geometry of visual space than the two-dimensional rectilinear constraints. To the end of exploring the idealized geometry, tests have been carried out in which the two-dimensional constraints have been shifted in position, e.g., to the four corners of a rhombus, or have been interleaved, e.g., to the four corners of the square, $(1,1)(1,2)(3,1)(3,2)$. Comparable operations were imposed upon one-dimensional constraints. Performance was uniformly poorer under these conditions than with the methods employed here, especially with the two-dimensional constraints. Thus, while the visual system probably does not process spatial information in the manner described for imposing Markov constraints, better procedures may not be available for displaying such constraints spatially.

\section{REFERENCES}

GIBSON, J. J. The perception of the visual world. Boston: Houghton-Mifflin, 1950.

JULESZ, B. Visual pattern discrimination. Institute of Radio Engineers Transactions on Information Theory, 1962, IT-8, 84-92.

PICKETT, R. S. The perception of a visual texture. Journal of Experimental Psychology, $1964,68,13-20$.

\section{NOTE}

1. Professor D. Krantz has kindly pointed out a simplification in the conceptualization of the random generating process. Instead of generating both the top row and first column at random. consider very long "infinite" horizontal displays. The first column of each line is determined by the last element of the previous line. This entry will be independent of the first element of the previous line. This leads to a picture of the random generating process as a single string, rather than as a series of strings with independent starting states.

(Accepted for publication October 17, 1970.) 